\title{
Transformation and Innovation of Human Resource Management in the Era of Big Data under the Background of Industry Intelligence
}

\author{
Shuchen Liu \\ Department of Human Resource, Southeast University, Nanjing, Jiangsu, China
}

\begin{abstract}
In the context of industry intelligence, modernization in management has achieved remarkable advance. Management concepts and management methods are two key factors in determining the effects of human resource management (HRM). The application of big data in HRM not only drives the modernization of management concepts, but also promotes the innovation of management methods, making the management work better match the needs of the organization's operation. This paper explores how human resource management is delicately conducted in an information-based and intelligent society in terms of the shift in management concepts, the optimization of the flow of the management, the benefits of innovation in management. In the meantime, corresponding strategies and suggestions on how to bring full play to the positive effects of big data in HRM are put forward to meet the needs of modern management goals.
\end{abstract}

Keywords: big data, industry intelligence, human resource management (HRM), change, innovation

\section{Introduction}

As modernization of an organization speeds up in a rapidly changing society, the spectrum and degree of the application of big data technologies in the management work is dramatically expanded. In this case, how human resource management, as the fundamental element in the managerial area, integrates with big data technologies is becoming all the more important. It turns out that big data technologies not only help operate all kinds of information scientifically and efficiently, but also provides sound grounds for the decision-making processes, contributing to the development of an organization[1]. At the same time, the application of big data puts forward higher technical and management requirements for HRM, which requires organizations to carry out targeted analysis and research according to the actual situation of their own management, develop more functions suitable for the actual HRM, and meet the needs of the development of HRM in the era of big data. Currently, with the shift from the traditional matter-focused approach to the strategic approach, there have been profound changes and innovations in the concepts, methods and effects of HRM. Therefore, it is necessary for us to understand new contents and characteristics of HRM and take constructive and effective measures to promote the high-quality development of management work.

\section{Changes in the Management Concepts}

Management concepts refer to thoughts and ideas guiding the operation of an organization. Nowadays, the highly advanced information technology not only provides a strong support for the choice of ways in management, but also makes management concepts more complicated. For example, big data may cause some organizations to lose track of direction and miss the opportunity for development due to misconception. It is widely accepted that the changes of concepts tend to experience a gradual process, which will be reinforced with the passage of time. There are at least 3 major changes which have taken shape or are taking place as in the following Table 1.

Table 1 Changes in the management concepts

\begin{tabular}{|c|c|c|}
\hline Traditional HRM & & HRM in the era of big data \\
\hline Process-centered & TO & Data-centered \\
\hline
\end{tabular}

ISSN: 0010-8189

(c) CONVERTER 2020

www.converter-magazine.info 


\begin{tabular}{|c|c|c|}
\hline Small samples & TO & Full samples \\
\hline Causation & TO & Correlation \\
\hline
\end{tabular}

\subsection{From process-centered to data-centered}

The traditional HRM mode is centered on "matters" which the management departments and staff divide into several steps and stages to ensure the operation of planned tasks and achieve the expected goals by keeping an eye on the flow of tasks. While this old management concept may lead to the accuracy and stability of the work process, it hardly fits into the work handling large amounts of matters.

In the era of big data, the concept of HRM has gradually shifted to data as the core, that is, how to acquire data, how to dig and realize the value of data and seek strategies for optimization in practice. As in personnel management, for instance, HR department can precisely predict the employees' needs through Regression Analysis, check the equality of salaries through collected data, comprehensively review workers behaviors and performances through Staff Competency Models, predict probability of dismission through Boosting and Random Forests and reads employees' letters of resignation through Affective Analysis etc. Evidently, the management mode in the big data era has surpassed the traditional one with the flow of process as the core, and transformed into a new management model centered on data, which optimizes the process and effects of HRM and fits into the requirements of the big data era[2].

This transformation has implications for the development of HRM. While dealing with problems, we should think in the way with data as the core; while acquiring as much data as possible, we should dig out what lie beneath the data and make them serve the management work.

\subsection{From small samples to full samples}

Sample size is the key index determining whether a decision is scientific and correct. Previously, information data were presented in the form of paper planning books, design drawings, and reports, etc. Although it can provide relatively rich information, its defects in data integration and retrieval greatly limit the sample size of HRM[3]. Besides, for large-sized organizations, HR departments inclined to adopt and apply small samples to save costs, which would in turn cause narrow-mindedness and single-mindedness on the management part. Conclusions drawn from such sampling turned out to be untrustworthy and unreliable.

The desire to mine as much data as possible drives the management departments and staff to reach for all data samples and summarize rules and laws so as to make more accurate judgments on uncertain factors and finally make reliable decisions. For example, instead of being operated carelessly, recruitment should be conducted with precision; wage adjustment plans should not be conducted based on small samples but measured with full size samples, in which case, opinions should be collected from management sectors as well as from as many workers as possible, through online polls for instance, to ensure a scientific and effective operating system.

In terms of the growth of an organization, the change in the size of sampling reveals the instability in operating systems within this organization and calls attention to the corresponding measures, that is, effectively enlarge the sample volume, through which large amounts of data can be collected. According to statics, the larger the data, the bigger the probability to find out patterns.

\subsection{From causation to correlation}

HRM work is a service by nature. In addition to paying attention to the results of the management, we should also explore the resulting causes for solving problems and drawing general laws. This concept guided by causal relationship has long been the key thinking on which the HRM relies[4]. In light of this concept, in order to prevent a decision which would exert negative effects in the future, these negative effects will be predicted by way of

ISSN: 0010-8189 
hypothesis and reasoning so as to provide references for decision making. This causation-based concept may reduce operating risks, but chances are that it will cause misjudgments and wrong decisions.

In the era of big data, HRM work focuses more on studying the correlation among variables. Through collecting and analyzing large quantity of data, various hypothesis and models attained through analysis of correlation among variables can be verified and underlying rules and laws can be obtained, providing a more sound and reliable ground for making decisions. In contrast to causation, correlation incorporates all the possible factors that might influence a certain decision in a common system, make bold predictions about the correlativity and conduct detailed verification via bulk data, thus ensuring the advancement and foresight in management. A case in point is the issue about employees' departure. While the employees may claim that they provide reasons for departure, these reasons are not real ones deep down. With the help of data analysis, judging from the letters of resignation and the employees work performance, related variables and their correlation with resignation can be systematically sorted out. In this way, the management over issues regarding workers turnover can be improved.

In the current time when many uncertainties occur, the traditional paradigm formed on the basis of causation has gradually lost its advantage, while the new model based on the analysis of correlation among data are more suitable when a decision is about to be made. Therefore, the concept of correlation analysis should be recognized and a large number of resources should be invested to conduct correlation analysis.

\section{The Optimization in the Flow of Process}

With many more uncertainties coming up, an increasing number of attentions has been placed on the whole process management, hoping to ensure the smooth realization of the final result through the fine management of each small step. In the big data era, with the application and popularization of computer technology, the whole process of HRM has been optimized, which is vital in speeding up modernization in management. There are mainly 4 shifts as seen in the following Table 2 .

Table 2 The optimization in the flow of process

\begin{tabular}{|c|c|c|}
\hline Traditional HRM & & HRM in the era of big data \\
\hline Paper files & TO & Digital and paperless files \\
\hline Qualitative analysis & TO & $\begin{array}{c}\text { Combination of qualitative and } \\
\text { quantitative analysis }\end{array}$ \\
\hline Modeling-based prediction & TO & Intelligent decision \\
\hline Manual operation & TO & Machine operation \\
\hline
\end{tabular}

3.1 A shift from paper files to digital and paperless files

The widespread use of digitalized management systems by modern organizations has greatly changed the way that HRM depends on to obtain data source. The traditional way to access HRM information through paper files and materials is gradually transformed into the digitalized management systems characterized by paperless operation. Thanks to the modern technology, a large number of data can be made electronic through scanning and goes into the human digitalized management system, making it possible to process data according to different functional options.

In contrast to the traditional paper files, the paperless operation not only saves the cost of data processing, but also avoids the impact of subjective errors on the results of data processing, making the HRM more scientific and effective.

3.2 A shift from qualitative analysis to the combination of qualitative and quantitative analysis

Although human resource management is the management of people's working ideas, contents and methods, and

ISSN: 0010-8189

(C) CONVERTER 2020

www.converter-magazine.info 
has the characteristics of qualitative management, the status and characteristics of the overall operation of human resource service organizations determine that all decisions cannot be made by qualitative analysis alone.

The combination of qualitative analysis with quantitative analysis is the distinct change and feature of HRM in the big data era, that is, in the process of data analysis and decision-making, quantitative analysis is applied to the collected data, inferences about the possible results of a decision is reached by means of correlation analysis and eventually several desired plans are formed to choose from. And then these plans will be analyzed by means of qualitative methods according to the internal and external factors facing the organizations, through which a final plan will be set up to fit the actual needs of the organization's operation[5].

Compared with the traditional qualitative analysis characterized by small sampling and collective negotiation, the means to combining qualitative and quantitative analysis under the big data mode is not only as scientific and accurate as data analysis-based quantitative analysis, but also takes into account of the humanization advantage possessed by qualitative analysis, making HRM more suitable for the development of modern organizations.

\subsection{A shift from modeling-based prediction to intelligent decision}

According to Bernard Marr, a specialist on big data analysis, the idea behind data-driven HRM is to make HRM more intelligent by various possible means.

Compared with traditional model predictive method system based on various assumptions, big data-based predictive models fully assimilate and perform the core function of big data on prediction by applying algorithm to the substantive data and predicting the possibilities of things that might happen. Not only can we predict a lot of things in this way, but we can also make the predictions deeper. Besides, the predictive function of big data is realized on the basis of the collection of a large number of data related to specific objects. This special data provides more personalized options available for data prediction, goes beyond the previous robot-type model in terms of algorithm and effect, and endows big data with the attribute of artificial intelligence.

For example, in the process of decision-making, in addition to proposing relevant decision-making schemes based on various data collected, the big data system will also recommend preferential decision-making schemes based on the previous decision preferences of decision-makers, and make the management work more humane and intelligent owing to the advantages of the schemes.

\subsection{A shift from manual operation to machine operation}

The traditional HRM is more dependent on the manual operation on the part of HRM staff, and the content of management is mainly presented in the form of written reports and forms, which results in the low efficiency on information retrieval and utilization.

In the era of big data, HRM data are able to be conducted through an informalized management system, in which related information is recorded into the HRM system through robot data terminals, then the system will implement classified management and quantitative analysis of data, and finally deliver reliable results. This artificial intelligence management mode not only improves the reliability of data information management, but also increases the efficiency and effect of data utilization, making human resource management more accurate and effective.

\section{Innovation of HRM Effects in the Era of Big Data}

In the big data era, data can be utilized for different purposes by any organizations. Generally speaking, the purposes fall into four categories: making make better decisions, better understanding customer needs, improving operations, and realizing monetization of data. For HRM practices, the purposes of using data are mainly reflected

ISSN: 0010-8189

(C) CONVERTER 2020 
in the first three categories, which are precisely the areas which the innovations of HRM effects lie in. According to the findings of research, the innovative outcomes of modern HRM manifest themselves in many aspects with some seen from macro or micro level, some other seen from general or local level and still those with short-term or long-term effects. The 3 major transformations are listed in the following Table 3.

Table 3 Innovation of HRM effects in the era of big data

\begin{tabular}{|c|c|c|}
\hline Traditional HRM & & HRM in the era of big data \\
\hline Static & TO & Dynamic \\
\hline Vagueness & TO & Precision \\
\hline Low quality and low efficiency & TO & High quality and high efficiency \\
\hline
\end{tabular}

\subsection{Transformation from static to dynamic}

With the wide application of big data technologies, the digital HRM system can not only automatically design and select intelligent data processing methods, but also deliver the predicted results under different data processing schemes, so as to provide strong support for the enrichment of the effect of human resource management[6]. At the same time, with the continuous increase of the amount of data received, the system can carry out intelligent update and improvement on its own, matching the data processing process and data processing content, and then produce results in line with the actual situation. As a result, what people often say as "dynamic management, real-time monitoring" has become a reality in the daily practices of HRM.

Compared with the intelligence of HRM methods, the intelligence of process and results is the highlight of the application of big data in human resource management.

\subsection{Transformation from vagueness to precision}

HRM is a systematic and complex task. In addition to being affected by fixed factors, it may also be affected by a large number of uncertain variables, which can exert greater influence. In light of this, there is a request that results of HRM be highly matched with actual demands, providing both macro trends and micro details.

Based on the big data, HRM can not only predict and test the correlation of data variables, but also produce more accurate results through optimization of algorithms to meet the needs of detailed information. The advantages of combining correlation and precision together in the big data era lie in that it not only meets the needs of trend analysis and details grasping, but also creates good conditions for a scientific, comprehensive and accurate decision-making process, facilitating the high-quality development of HRM.

Take the employee's salary fairness evaluation as an example. It is possible to grasp the disparity in salaries and benefits among employees of different abilities by quantitatively analyzing the salary data in a certain period of time in the past, or to analyze the key factors relevant to employees' salaries and benefits through the corresponding salary evaluation model. The combination of the correlation and accuracy enhances the effectiveness of human resources management.

\subsection{Transformation from low quality and low efficiency to high quality and high efficiency}

In the development of modern organizations, the flood of uncertain factors makes the time, money and risk costs of HRM generally increase, and the variability of organizational environment makes the efficiency of decisionmaking activities more critical. The contradiction of high cost and low efficiency which makes the low cost and high efficiency this pair of contradictions be clearly reflected in the management work, and for the sake of cost, human resource management problems of inferior quality and low for a long time.

In the era of big data, the application of a digitalized information system has greatly reduced the various costs of

ISSN: 0010-8189 
data information acquisition and processing, and also eliminated the risk factors in decision-making, so that the cost of human resource management has been greatly reduced. At the same time, in terms of efficiency, the correlation analysis in the big data era simplifies the analysis process of what $\rightarrow$ why $\rightarrow$ how to do on the basis of causal relationship analysis to what $\rightarrow$ how to do it, so that the management process does not require too much attention. The question of "why" is discarded, thereby improving the efficiency of human resource management[7].

\section{Conclusion}

As organizational operations have entered the era of big data, how to obtain and use batch data information has gradually become a key content in organizational management under the background of industry intelligence. As far as human resource management is concerned, the application of big data not only changes the original management concept, but also realizes the role of changing management methods and enriching management effects, thereby promoting the smooth development of modern human resource management. With rapid development and increasingly fierce competition, it is necessary to grasp the relevant content and laws of human resource management reform and innovation, and leverage big data to broaden human resource management ideas, optimize human resource management methods, the modern development of human resource management.

\section{References}

[1] M.X. Shang, "The transformation of enterprise human resource management in the era of big data," Modern Business and Trade Industry, vol. 08, pp. 71-72, 2019.

[2] Z. Cai, "Human resource management in the era of big data," Beijing: Tsinghua University Press, 2016.

[3] X.L. Pan, "Human Resource Management Information System Based on Cloud Computing," Electronic Technology and Software Engineering, vol. 05, pp. 152, 2019.

[4] X.Y. Li, "Analysis of the application value of big data in human resource management," Enterprise Reform and Management, vol. 05, pp. 66-67, 2019.

[5] T. Zhang, "Research on Human Resources Management in the Big Data Era," Shanxi Agricultural Economics, vol. 04, pp. 155+157, 2019.

[6] X.L. Hu, "Research on Enterprise Human Resource Management Innovation under Big Data," Economist, vol. 04, pp. 264-265, 2019.

[7] B. Mar, "Human Resource Data Analysis Human Resource Management in the Era of Artificial Intelligence," Beijing: Machinery Industry Press, 2019. 